\title{
Inconsistency in reporting potentially traumatic
}

\section{events}

\author{
URS HEPP, ALEX GAMMA, GABRIELLA MILOS, DOMINIQUE EICH, \\ VLADETA AJDACIC-GROSS, WULF RÖSSLER, JULES ANGST \\ and ULRICH SCHNYDER
}

\author{
Background Research on post- \\ traumatic stress disorder (PTSD) relies \\ mainly on self-reports of exposure to \\ trauma and its consequences.
}

\begin{abstract}
Aims To analyse the consistency of the reporting of potentially traumatic events (PTEs) over time.
\end{abstract}

Method Acommunity-based cohort, representative of the canton of Zurich,

Switzerland, was interviewed at the ages of 34-35 years (in 1993) and 40-4l years (in 1999). A semi-structured diagnostic interview, including a section on PTSD, was administered.

Results Of the 342 participants who attended both interviews, 169 reported some PTE (1993, $n=110 ; 1999, n=120)$. In 1999, 56 participants (33.1\%) reported for the first time PTEs that actually occurred before 1993, but which had not been reported in the 1993 interview. In total, 68 participants (40.2\%) who had reported a PTE in 1993 did not report it in 1999. The overall frequency of inconsistent reporting was $63.9 \%$.

\section{Conclusions The high level of} inconsistency in the reporting of PTEs has implications for therapy as well as for research.

\section{Declaration of interest None.}

Funding detailed in Acknowledgements.
Most research on trauma and its consequences for mental health relies on self-reports of potentially traumatic events (PTEs). However, self-reports could be biased for a variety of reasons. An underestimation of the frequency of PTEs could result from the trauma being repressed, dissociated or simply forgotten. Furthermore, the victims of trauma might feel ashamed about PTEs, and therefore might not be prepared to disclose them. Research into the consistency of reports of PTEs has so far mainly focused on highly selective samples, such as individuals who suffered childhood sexual abuse (Williams, 1994; Widom \& Morris, 1997; Goodman et al, 2003) or combat-related experiences (Southwick et al, 1997; Roemer et al, 1998). To our knowledge, the consistency of reports of PTEs in the general population has never been systematically investigated. The aim of the present study was to analyse the consistency of reporting of PTEs over time in a large and representative community-based sample.

\section{METHOD}

\section{Sample}

The initial screening sample of the Zurich Cohort Study in 1978 consisted of a cohort of 4547 young adults (2201 men aged 19 years and 2346 women aged 20 years), representative of the canton of Zurich, Switzerland. Participants were screened by means of the Symptom Checklist 90 Revised (SCL-90-R; Derogatis, 1986) in 1978. From this cohort a sub-sample of 591 participants $(292$ men and 299 women) was drawn. Two-thirds of the sample were randomly selected from those scoring above the 85 th percentile on the SCL-90-R, and one-third was randomly selected from those scoring below the 85 th percentile.

Between 1979 and 1999 all of the participants were prospectively interviewed six times. For the purposes of the present study, data from the initial 1978 screening and from the 1993 and 1999 interviews were further analysed. In 1993 a total of 407 participants (192 men and 215 women) and in 1999 a total of 367 participants (162 men and 205 women) were interviewed. Altogether 342 individuals (151 men and 191 women) attended both interviews (in 1993 and 1999). Drop-outs from the 1999 interview were less likely to be female $(P=0.001)$. In the 1993 and 1999 interviews drop-outs were more often single (1993, $P<0.001$; 1999, $P<0.001)$. Dropouts from both interviews had less lifetime psychiatric comorbidity $(P<0.001)$, but did not differ significantly with regard to the SCL-90 assessed in 1978. Further methodological details of the study have been described elsewhere (Angst et al, 1997; Eich et al, 2003; Merikangas et al, 2003).

\section{Measures}

The Structured Psychopathological Interview and Rating of the Social Consequences for Epidemiology (SPIKE; Angst \& Dobler-Mikola, 1985) was administered by clinically trained psychiatric residents and clinical psychologists. For the 1993 interview the SPIKE was complemented by a PTSD module according to the DSM-III-R (American Psychiatric Association, 1987). For the 1999 investigation the PTSD section of the interview was adapted to the DSM-IV (American Psychiatric Association, 1994). Information about PTEs was obtained by asking the participants whether they had experienced or witnessed an event or events that involved actual death of others or threatened death, serious injury or a threat to the physical integrity of self or others (criterion A1 for PTSD). Several typical events were proposed, and participants were asked whether they had experienced any of these or similar events. In the 1999 interview, participants were also asked whether they felt intense fear, helplessness or horror (criterion A2 for PTSD according to DSM-IV). Participants could report multiple events. In addition, they were asked about the presence of PTSD symptoms (criteria B, C and D) and their duration (criterion $\mathrm{E}$ ), and whether any distress and/or impairment in social, occupational or other important areas of functioning had been caused by the disturbance (criterion F).

Participants who reported exposure to a PTE were asked to describe the trauma. 
PTEs were categorised according to Breslau et al (1998) as follows: 1, assaultive violence; 2, any other injury or shocking experience; 3 , learning about traumas experienced by others; 4 , sudden unexpected death of a close friend or relative.

Two types of inconsistent reporting of PTE were defined: Type I inconsistency was defined as a PTE that occurred before the 1993 interview and was reported in 1999 but not in 1993. Type II inconsistency was defined as a PTE that occurred before the 1993 interview and was reported in 1993 but not in 1999. According to these definitions a combination of Type I and Type II inconsistency in the same individual was possible. PTEs that occurred before 1993 and were reported in both 1993 and 1999 constituted the main type of consistent reports. Events that were reported in 1999 and which occurred between the 1993 and 1999 interviews (and were therefore not reported in 1993) were also defined as consistent reports.

The SCL-90-R (Derogatis, 1986; Franke, 1995) was used to assess a broad spectrum of psychological and somatic complaints. This instrument comprises nine sub-scales: somatisation, obsessivecompulsive disorder, interpersonal sensitivity, depression, anxiety, anger/hostility, phobia, paranoid ideation and psychoticism. Two independent SCL scales were also used, namely emotional lability and vegetative lability. These were derived from factor and cluster analyses of a sample of 5310 men and women from the canton of Zurich, which included the initial screening sample used for the Zurich study (Scheidegger, 1992). Emotional lability describes psychologically experienced complaints and includes items from all of the original SCL scales except those for somatisation and hostility. Vegetative lability describes functional somatic complaints and is mainly composed of items from the original somatisation scale.

The Freiburg Personality Inventory (FPI) is a 212-item self-rated questionnaire comprising nine primary personality factors (nervousness, spontaneous aggression, depressiveness, irritability, sociability, resilience, striving for dominance, inhibition and openness) (Fahrenberg et al, 1970). Three secondary factors (aggressiveness, extraversion and neuroticism/vegetative lability) were derived from a factor analysis of large samples, as described elsewhere (Angst \& Clayton, 1986; Scheidegger, 1992; Merikangas et al, 1993).
The 64-item Questionnaire on Modes of Coping with Chronic Disease was used to assess coping (Klauer, 1989). Self-esteem and mastery were assessed with the Structure of Coping Questionnaire (Pearlin \& Schooler, 1978).

A modified life-event inventory based on the scales developed by Tennant and Andrews (Tennant \& Andrews, 1976, 1977; Angst et al, 1984) was used to assess general life events. Items that were not applicable to young people (e.g. retirement) were omitted and some other more suitable items were added, to allow assessment of changes in life habits (Holmes \& Rahe, 1967).

\section{Statistical analyses}

Groups were compared using KruskalWallis tests for rank-ordered data, and chi-squared tests for frequencies. All analyses were performed using Stata 8.2. for Windows 2000.

The study was approved by the Institutional Review Board of the University of Zurich, and informed consent was obtained from all participants.

\section{RESULTS}

\section{Prevalence of exposure to PTEs}

In the 1993 interview, 130 (31.9\%) of the participants $(28.7 \%$ of the men and $34.9 \%$ of the women; $P=0.18$ ) reported a total of 147 PTEs. Of these, 115 participants $(88.5 \%)$ reported one event, 13 $(10.0 \%)$ reported two events and 2 $(1.5 \%)$ reported three or more events. The weighted lifetime prevalence of exposure to PTEs was $26.6 \%$ (95\% CI 20.8-33.4). It was $21.7 \%(95 \%$ CI $14.5-31.2)$ for men and $31.4 \%(95 \%$ CI $22.8-41.5)$ for women $(P=0.13)$.

In the 1999 interview, 128 (34.9\%) of the participants $(33.3 \%$ of the men and $36.1 \%$ of the women; $P=0.58$ ) reported 179 lifetime PTEs. Of these, 91 participants $(71.1 \%)$ reported one event, $23(18.0 \%)$ reported two events and 14 (10.9\%) reported three events. This corresponds to a weighted lifetime prevalence of exposure to PTEs of $28.0 \%$ (95\% CI $21.8-35.2$ ), with values of $27.5 \%$ (95\% CI 18.7-38.3) for men and $28.5 \%$ (95\% CI 20.3-38.4) for women $(P=0.88)$.

At both investigations women reported more assaultive violence, especially sexual assault (both $P<0.001$ ), than men. This difference did not remain significant when the prevalence rates were weighted for the stratified sample.

\section{Inconsistent reports of PTE}

Of the 342 participants who attended both interviews over a time span of 6 years, 169 reported exposure to PTEs (110 participants in 1993 and 120 participants in 1999). In 1999, 56 participants (33.1\%) reported for the first time PTEs that had occurred before the 1993 interview but which were not reported in 1993 (Type I inconsistency). A total of 68 participants (40.2\%) who had reported a PTE in 1993 no longer reported it in 1999 (Type II inconsistency). Overall, there was some inconsistent reporting (Type I and/or Type II inconsistency) in $108(63.9 \%)$ of the 169 participants who reported a PTE. Of the participants who reported assaultive and sexual PTEs in 1993, 52.6\% were prospectively inconsistent, compared with $67.2 \%$ of the participants who reported non-assaultive, non-sexual types of PTE $(P=0.1)$. In total, $29.0 \%$ of the participants who reported assaultive or sexual PTEs in 1999 were retrospectively inconsistent, whereas $71.7 \%$ of the participants who reported no assaultive or sexual PTEs in 1999 were inconsistent $(P<0.001)$. In 1999 , the proportion of participants who had been exposed to assaultive or sexual violence was significantly higher in consistent reporters than in inconsistent reporters $(36.1 \%$ v. $8.3 \% ; P=0.0001)$, and this tendency was already apparent in 1993 (29.5\% v. $18.5 \% ; P=0.10)$.

The data for consistent and inconsistent reports of PTEs are shown in Table 1. The proportions of the four categories of PTE cited by consistent and inconsistent reporters are shown in Table 2.

\section{Group comparisons between consistent and inconsistent reporters}

There was a trend towards more consistent reporting of PTEs by women than by men (41.1\% v. 28.6\%; $P=0.087)$. This effect was mainly due to a greater proportion of men with a Type $\mathrm{I}$ inconsistency $(P=$ 0.054). There were no differences between consistent and inconsistent reporters with regard to social class and level of education as assessed in 1979, FPI score (assessed in 1993 and 1999), or mastery and coping (assessed in 1993 and 1999). The only difference was found for self-esteem in 1999, with inconsistent reporters scoring 
Table I Consistent and inconsistent reports of potentially traumatic events (PTEs) in 1993 and 1999

\begin{tabular}{|c|c|c|c|c|c|}
\hline & CR & $\begin{array}{c}\text { Type I } \\
\text { IR }\end{array}$ & $\begin{array}{c}\text { Type II } \\
\text { IR }\end{array}$ & $\begin{array}{c}\text { Type I/II } \\
\text { IR }\end{array}$ & Tota \\
\hline \multicolumn{6}{|c|}{ Report of any PTE in 1993} \\
\hline No & 191 & 40 & 0 & 0 & 59 \\
\hline Yes & 42 & 0 & 52 & $16^{2}$ & 110 \\
\hline Total & 61 & 40 & 52 & 16 & 169 \\
\hline \multicolumn{6}{|c|}{ Report of any PTE in 1999} \\
\hline No & 0 & 0 & 49 & 0 & 49 \\
\hline Yes & 61 & 40 & $3^{3}$ & $16^{2}$ & 120 \\
\hline Total & 61 & 40 & 52 & 16 & 169 \\
\hline
\end{tabular}

CR, consistent reporting; Type I IR, Type I inconsistent reporting (PTE occurring before 1993, and reported in I999 but not in 1993); Type II IR, Type II inconsistent reporting (PTE occurring before 1993, and reported in 1993 but not in 1999); Type $I / I I I$, individuals showing both types of inconsistency.

I. Participants who reported in 1999 any PTE that occurred after the 1993 interview and was therefore not reported in 1993.

2. Participants who reported a PTE in 1993 but not in 1999 (Type II IR), and who reported another PTE in 1999 but not in 1993 (Type I IR).

3. Participants who reported a PTE in 1993 but not in 1999 (Type II IR), and who reported another PTE in 1999 that occurred between the two interviews (consistent reporting).

Table 2 Frequency of the different potentially traumatic event (PTE) categories in consistent and inconsistent reporters

\begin{tabular}{|c|c|c|c|c|c|c|}
\hline \multirow[b]{2}{*}{ PTE category } & \multicolumn{3}{|c|}{1993} & \multicolumn{3}{|c|}{1999} \\
\hline & $\begin{array}{l}\text { Consistent } \\
\text { reporters } \\
(n=6 \mathrm{I})\end{array}$ & $\begin{array}{l}\text { Inconsistent } \\
\text { reporters } \\
(n=108)\end{array}$ & $P\left(\chi^{2}\right)$ & $\begin{array}{c}\text { Consistent } \\
\text { reporters } \\
(n=61)\end{array}$ & $\begin{array}{l}\text { Inconsistent } \\
\text { reporters } \\
(n=108)\end{array}$ & $P\left(\chi^{2}\right)$ \\
\hline Assaultive violence & 29.5 & 18.5 & 0.10 & 36.1 & 8.3 & 0.0001 \\
\hline $\begin{array}{l}\text { Other injury or other } \\
\text { shocking experience }\end{array}$ & 27.9 & 25.9 & 0.78 & 47.5 & 32.4 & 0.06 \\
\hline $\begin{array}{l}\text { Learning about traumas } \\
\text { experienced by others }\end{array}$ & 1.6 & 5.6 & 0.22 & 8.2 & 3.7 & 0.21 \\
\hline $\begin{array}{l}\text { Sudden unexpected } \\
\text { death (or threat of death) } \\
\text { of close friend or relative }\end{array}$ & II.5 & 15.7 & 0.45 & 24.6 & 18.5 & 0.35 \\
\hline
\end{tabular}

higher on this sub-scale of the Structure of Coping Questionnaire (14.77 v. 15.79; Kruskal-Wallis test, $P=0.0045)$. In 1993 a higher proportion of consistent reporters (34.4\% v. $12.8 \% ; P=0.001)$ acknowledged having memory problems (scoring $>2$ on the SCL-90-R memory item 9). In 1999 this difference was no longer significant. The FPI openness sub-scale, which is a measure of dissimulation, revealed no significant group differences, and neither did any of the three secondary factors, namely aggressiveness, extraversion and neuroticism/vegetative lability.

The mean number of PTSD symptoms was higher in consistent than in inconsistent reporters of PTEs (1999: $1.51 v$. $0.56, \quad P=0.01 ; \quad 1993: 1.28 \quad v . \quad 0.55$,
$P=0.08)$. There was a trend towards more re-experiencing of the trauma by consistent reporters than by inconsistent ones (1999: $P=0.052$; 1993: $P=0.14)$. In 1993, a slightly higher proportion of consistent reporters than of inconsistent ones felt a threat to their own physical existence or that of someone close to them during the PTE. However, this difference was not significant $(59.0 \% \quad v .48 .6 \% ; P=0.19)$. In 1999, significantly more consistent reporters felt intense fear, helplessness or horror during the PTE $(90.2 \% v .44 .3 \%$; $P<0.001)$.

There was no difference between consistent and inconsistent reporters in the proportion of participants with lifetime major depressive episodes $(45.4 \% \quad v$.
$45.9 \% ; P=0.95)$. No differences were found when taking into account lifetime sub-threshold mood syndromes (minor depression, recurrent brief depression, dysthymia) or depressive symptoms $(P=0.21)$. Similarly, the SCL-90-R depression, anxiety and somatisation scores at age 19-20 years (in 1978) showed no difference between the two groups.

\section{DISCUSSION}

Memory of traumatic events is widely discussed in the field of psychotraumatology. Indeed, memory is a crucial issue in posttraumatic stress disorder (PTSD). On the one hand, a core symptom of PTSD according to DSM-IV (American Psychiatric Association, 1994) is the re-experiencing of the traumatic event in the form of intrusive and distressing recollection of the event or flashbacks (criterion B). On the other hand, there may be avoidance of thoughts and feelings associated with the trauma, and inability to recall important aspects of the event (criterion C). To date, systematic research into the consistency of reports of PTEs has mainly focused on survivors of childhood sexual abuse and military veterans. To the best of our knowledge, this is the first representative community-based study that has investigated the prevalence and consistency of reported exposure to PTEs over time.

\section{Limitations of the study}

Several limitations of this study need to be addressed. First, it relies on information about PTEs obtained during interviews, and no objective data are available. A possible confounding effect could stem from the change from DSM-III-R (American Psychiatric Association, 1987) to DSM-IV (American Psychiatric Association, 1994) between the two interviews. The DSMIII-R included the stressor criterion that refers to the aetiological event as 'a psychologically traumatic event that is generally outside the range of usual human experience', such as war combat experience, natural disaster or criminal assault. The diagnostic criteria were revised in the DSM-IV (American Psychiatric Association, 1994). The symptoms of PTSD have changed little, but the stressor criterion has been extended to a broader range of PTEs. In the 1993 interview participants were asked about exposure to PTEs according to the DSM-III-R, but in 
addition the interviewers asked whether they had experienced any other PTE (e.g. motor vehicle accident). The coding of each event according to Breslau et al (1998) for the 1993 and 1999 interviews, with exclusion of events that did not fulfil the DSMIV criterion A1 (e.g. parent's divorce, father's bankruptcy), provided sufficient comparability of the reports of PTEs. A further limitation of the study is the overall low frequency of PTSD symptoms in our sample, which restricts further analysis of consistency with regard to severity of PTSD.

\section{Prevalence of exposure to PTEs}

Compared with data from international epidemiological studies, the PTE prevalence rates in our sample are relatively low (Kessler et al, 1995; Stein et al, 1997; Breslau et al, 1998; Creamer et al, 2001). Women reported significantly more exposure to assaultive and sexual violence than did men. The great majority of the participants who reported any exposure to PTEs reported a single event, which may partly explain the low prevalence of PTSD symptoms.

\section{Inconsistent reports of PTEs}

Despite the relatively low exposure rate, the study participants reported a wide spectrum of PTEs, and because of the large size of the sample, trauma types that are relatively rare in the general population could be observed. Two types of inconsistent reporting of PTE exposure occurred. One in three participants who in 1999 reported a PTE that took place before the 1993 interview had not reported this event in the 1993 interview (Type I inconsistency). In total, $40 \%$ of the participants who reported exposure to a PTE in the 1993 interview did not report this event 6 years later (Type II inconsistency). Overall, among almost two-thirds of all participants who reported any exposure to traumatic events there was some inconsistent reporting of PTEs. This demonstrates the high level of instability of reports of exposure to PTEs.

\section{Type of PTE and PTSD symptoms}

Although socio-demographic characteristics and personality traits did not explain inconsistent reports of PTEs, the type of trauma, the subjective perception of the PTE and the specific post-traumatic symptoms seem to partly explain the differences in consistency. Exposure to assaultive and sexual violence was reported more consistently (statistically significantly in 1999, and showing a trend in 1993). However, even among participants who had been exposed to assaultive or sexual violence, inconsistent reports were frequent. The trend for women to make more consistent reports could be explained by the higher level of exposure of women in this sample to assaultive and sexual violence. The mean number of PTSD symptoms was higher in consistently reporting participants, and we found re-experiencing as a specific posttraumatisation phenomenon that possibly enhances traumatic memory (Southwick $e t$ al, 1997; Roemer et al, 1998) to be slightly more frequent in consistently reporting participants. However, PTSD symptoms were also more frequent in participants who reported exposure to assaultive violence. The higher conditional risk of PTSD symptoms following assaultive or sexual violence compared with other PTEs is well documented (Breslau et al, 1998). Consistent reporters met the DSM-IV criterion A2, namely experiencing intense fear, helplessness or horror, more often than did inconsistent reporters. This result is compatible with previous findings (Krinsley et al, 2003).This emphasises the importance of the subjective reaction to PTEs.

The higher mean number of PTSD symptoms in consistent reporters is in agreement with previous findings in combat veterans. Retrospective accounts of warzone exposure showed an increase in the number of reported events over time that was correlated with the severity of PTSD symptoms, particularly intrusive symptoms (Roemer et al, 1998). In a prospective investigation of memory in veterans (Southwick et al, 1997), the severity of PTSD symptoms after 2 years was found to be correlated with overall inconsistency of memory. The authors of that study concluded that participants with higher levels of PTSD symptoms tend to amplify their memory of traumatic experience. However, given the overall low frequency of PTSD symptoms in our study, these results should not be over-interpreted.

\section{Traumatic memory}

The theory of dual representation of traumatic memory (Brewin et al, 1996) postulates that there is a verbally accessible memory system whereby traumatic events are integrated with other autobiographical memories and can deliberately be retrieved when required. In contrast, there is a situationally accessible memory system that does not use a verbal code, and which is triggered involuntarily by situational and emotional reminders of the trauma as it occurs (e.g. in flashbacks). A dissociated memory (i.e. better representation of the trauma information in one system than in the other) may partially explain inconsistent recall. Therefore whether or not an event is remembered might also depend on the actual state of emotion or on internal and external triggers.

However, a major cause of inconsistent reporting might well be simple oblivion. In a pre-study of the Zurich Cohort Study, the recall of behavioural and/or psychological problems documented in the school health records turned out to be poor (Kaeser, 1979; Weber, 1979). About a quarter of those with documented problems did not remember or report them in a questionnaire at the age of 20 years, showing the limited reliability of the information obtained at this young age, even though one would not expect to encounter memory problems at such an age. There was no difference in recall of behavioural compared with psychological problems. In our study, however, self-reported memory problems were not more frequent in inconsistent reporters, which does not support the idea that poor memory performance is a cause of inconsistent reports.

In long-term follow-up studies of children with a forensic or medical record of childhood sexual abuse, the proportion of victims of childhood sexual abuse who did not disclose the abuse ranges from 19\% to $38 \%$ (Williams, 1994; Widom \& Morris, 1997; Goodman et al, 2003). Delayed recall of traumatic experiences in childhood sexual abuse is not uncommon (Williams, 1995). In a representative communitybased sample, Elliott (1997) found a prevalence of some form of childhood PTE of $72 \%$. Retrospectively, about a third of the participants reported a partial or complete memory loss for the PTE for some time. Delayed recall was observed across various types of trauma, but was more likely if there had been interpersonal victimisation.

The interaction between the interviewer and the participant may be an additional source of inconsistency, because it might influence the participant's decision to disclose a PTE. Some participants may find it difficult to answer questions about traumatic events in a single interview. Even in 
the context of a therapy, a therapeutic alliance and confidence in the therapist must first be established before there is disclosure of traumatic experiences, especially interpersonal victimisation and childhood sexual abuse.

Our results cannot provide a simple explanation for the high level of inconsistency across different types of PTE. Exposure to violent situations seems to be more easily remembered and recalled, especially when it was associated with intense fear and helplessness and when it was followed by PTSD symptoms. However, the rate of inconsistency in participants who experienced assaultive or sexual PTEs is notable as well. Although it is easy to understand how a minor PTE such as an accident can be forgotten, it is more difficult to understand why a high proportion of participants 'forgot' a major PTE such as exposure to assaultive or sexual violence. Repression of PTEs, dissociation and recollection of lost memories may play a role, especially in severe trauma.

These findings are a challenge to research on the psychological consequences of trauma and to epidemiological research on exposure to PTEs and PTSD. Roemer et al (1997) stated that the field of traumatic stress too often treats retrospective reports as objective measures of exposure, despite the lack of evidence to support their accuracy. Further research into the consequences of exposure to PTEs should focus on prospective studies and/or rely on well-documented PTEs.

Although the findings from this community sample cannot be extrapolated to the reporting of severe trauma in clinical populations, there are some clinical implications. It is likely that in therapy, too, the reliability of an initial assessment of traumatic exposure is low, and that patients may not recall or disclose their traumatisation. The exposure to a PTE may only be recovered in the course of the therapy when a sustainable therapeutic alliance has been established.

\section{ACKNOWLEDGEMENT}

This work was supported by the Swiss National Science Foundation (grant 3200-050881.97/I).

\section{REFERENCES}

American Psychiatric Association (1987) Diagnostic and Statistical Manual of Mental Disorders (3rd edn, revised) (DSM-III-R). Washington, DC: APA.

\section{CLINICAL IMPLICATIONS}

Reports of exposure to potentially traumatic events (PTEs) are highly unreliable in a community sample across different types of trauma.

- Although the prevalence of exposure to PTEs was relatively low, exposure to some form of PTE in the general population is quite common.

- The low consistency of reported exposure to PTEs has to be taken into account in clinical practice as well as in research.

\section{LIMITATIONS}

- The study relies on information about exposure to PTEs that was obtained from the participants in interviews, and objective data were not available.

- The frequency of post-traumatic stress disorder (PTSD) symptoms in the sample was very low, limiting further analysis with regard to severity of PTSD symptoms.

- Between the two interviews the diagnostic criteria were changed from the DSMIII-R to the DSM-IV.

URS HEPP, MD, Department of Psychiatry, University Hospital Zurich; ALEX GAMMA, PhD, Psychiatric University Hospital Zurich; GABRIELLA MILOS, MD, Department of Psychiatry, University Hospital Zurich; DOMINIQUE EICH, MD, VLADETA AJDACIC-GROSS, PhD, WULF RÖSSLER, MD, JULES ANGST, MD, Psychiatric University Hospital Zurich; ULRICH SCHNYDER, MD, Department of Psychiatry, University Hospital Zurich, Zurich, Switzerland

Correspondence: Dr Urs Hepp, Department of Psychiatry, University Hospital Zurich, Culmannstrasse 8,CH-809l Zurich, Switzerland.Tel: +4l 44255 5280; e-mail: urshepp@bluewin.ch

(First received 22 December 2004, final revision 8 March 2005, accepted 22 March 2005)

American Psychiatric Association (1994) Diagnostic and Statistical Manual of Mental Disorders (4th edn) (DSM-IV). Washington, DC: APA.

Angst, J. \& Dobler-Mikola, A. (1985) The Zurich Study - a prospective epidemiological study of depressive, neurotic and psychosomatic syndromes. IV. Recurrent and nonrecurrent brief depression. European Archives of Psychiatry and Neurological Sciences, 234 408-416.

Angst, J. \& Clayton, P. (1986) Premorbid personality of depressive, bipolar and schizophrenic patients with special reference to suicidal issues. Comprehensive Psychiatry, 27, 51I-532.

Angst, J., Dobler-Mikola, A. \& Binder, J. (1984) The Zurich study - a prospective epidemiological study of depressive, neurotic and psychosomatic syndromes. I. Problem, methodology. European Archives of Psychiatry and Neurological Sciences, 234, 13-20.

Angst, J., Merikangas, K. R. \& Preisig, M. (1997) Subthreshold syndromes of depression and anxiety in the community. Journal of Clinical Psychiatry, 58, 6-10.

Breslau, N., Kessler, R. C., Chilcoat, H. D., et a (1998) Trauma and post-traumatic stress disorder in the community: the 1996 Detroit Area Survey of Trauma. Archives of General Psychiatry, 55, 626-632.
Brewin, C. R., Dalgleish, T. \& Joseph, S. (1996) A dual representation theory of post-traumatic stress disorder. Psychological Review, 103, 670-686.

Creamer, M., Burgess, P. \& McFarlane, A. C. (200I) Post-traumatic stress disorder: findings from the Australian National Survey of Mental Health and Wellbeing. Psychological Medicine, 31, 1237-1247.

Derogatis, L. R. (1986) $S C L-90-R$ : Administration, Scoring and Procedure Manual - II for the Revised Version. Townson, MD: Clinical Psychometric Research.

Eich, D., Ajdacic-Gross, V., Condrau, M., et al (2003) The Zurich Study: participation patterns and Symptom Checklist 90-R scores in six interviews, 1979-99. Acto Psychiatrica Scandinavica, 108 (suppl. 4I8), II-14.

Elliott, D. M. (1997) Traumatic events: prevalence and delayed recall in the general population. Journal of Consulting and Clinical Psychology, 65, 81I-820.

Fahrenberg, J., Selg, H. \& Hampel, R. (1970) Das Freiburger Persönlichkeitsinventar. Göttingen: Hogrefe.

Franke, G. (1995) SCL-90-R. Die Symptomcheckliste von Derogatis - Deutsche Version - Manual. Göttingen: Beltz Test.

Goodman, G. S., Ghetti, S., Quas, J. A., et al (2003) A prospective study of memory for child sexual abuse: new findings relevant to the repressed-memory controversy. Psychological Science, 14, I13-118. 
Holmes, T. H. \& Rahe, R. H. (1967) The Social Readjustment Rating Scale. Journal of Psychosomatic Research, II, 213-218.

Kaeser, D. (1979) Psychiatrische Katamnese von ehemaligen schulpsychiatrischen Fällen. Unpublished MD thesis. Faculty of Medicine, University of Zurich.

Kessler, R. C., Sonnega, A., Bromet, E., et al (1995) Post-traumatic stress disorder in the National Comorbidity Survey. Archives of General Psychiatry, 52, 1048-1060.

Klauer, T. (1989) Der 'Fragebogen zur Erfassung von Formen der Krankheitsbewältigung' (FEKB): Skalenkonstruktion und erste Befunde zu Reliabilität, Validität und Stabilität. Diagnostica, 35, 316-335.

Krinsley, K. E., Gallagher, J. G., Weathers, F. W., et al (2003) Consistency of retrospective reporting about exposure to traumatic events. Journal of Traumatic Stress, 16, 399-409.

Merikangas, K. R., Stevens, D. E. \& Angst, J. (1993) Headache and personality: results of a community sample of young adults. Journal of Psychiatric Research, 27, 187-196.

Merikangas, K. R., Zhang, H., Avenevoli, S., et al (2003) Longitudinal trajectories of depression and anxiety in a prospective community study: the Zurich Cohort Study. Archives of General Psychiatry, 60, 993-1000.

Pearlin, L. I. \& Schooler, C. (1978) The structure of coping. Journal of Health and Social Behavior, 19, 2-21.

\section{Roemer, L., Litz, B.T. \& Orsillo, S. M. (1997)}

Consistency of traumatic memories (letter). American Journal of Psychiatry, 154, 1628

Roemer, L., Litz, B. T., Orsillo, S. M., et al (1998) Increases in retrospective accounts of war-zone exposure over time: the role of PTSD symptom severity. Journal of Traumatic Stress, II, 597-605.

Scheidegger, P. (1992) Reproduzierbarkeit von Strukturen in empirischen Daten. Unpublished PhD thesis. Department of Psychology, University of Zurich.

Southwick, S. M., Morgan, C. A. 3rd, Nicolaou, A. L et al (1997) Consistency of memory for combat-related traumatic events in veterans of Operation Desert Storm. American Journal of Psychiatry, 154, 173-177.

Stein, M. B., Walker, J. R., Hazen, A. L., et al (1997) Full and partial post-traumatic stress disorder: findings from a community survey. American Journal of Psychiatry 154, III4-1119.
Tennant, C. \& Andrews, G. (1976) A scale to measure the stress of life events. Australian and New Zealand Journal of Psychiatry, 10, 27-32.

Tennant, C. \& Andrews, G. (1977) A scale to measure the cause of life events. Australian and New Zealand Journal of Psychiatry, II, 163-167.

Weber, M. (1979) Zuverlässigkeit der Beantwortung eines anamnestischen Fragebogens bei zwanzigjährigen Zürchern. Unpublished MD thesis. Faculty of Medicine, University of Zurich.

Widom, S. W. \& Morris, S. (1997) Accuracy of adult recollections of childhood victimization. Part 2 Childhood sexual abuse. Psychological Assessment, 9 34-46.

Williams, L. M. (1994) Recall of childhood trauma: a prospective study of women's memories of child sexual abuse. Journal of Consulting and Clinical Psychology, 62 , II67-1176.

Williams, L. M. (1995) Recovered memories of abuse in women with documented child sexual victimization histories. Journal of Traumatic Stress, 8, 649-673. 